Nikhef-2013-022

July, 2013

\title{
Nonlocal action for the super-Weyl anomalies: A new representation
}

\author{
Daniel Butter $^{a}$ and Sergei M. Kuzenko ${ }^{b}$ \\ ${ }^{a}$ Nikhef Theory Group \\ Science Park 105, 1098 XG Amsterdam, The Netherlands \\ dbutter@nikhef.nl \\ ${ }^{b}$ School of Physics M013, The University of Western Australia \\ 35 Stirling Highway, Crawley W.A. 6009, Australia
}

\begin{abstract}
Using the recently discovered $\mathcal{N}=1$ supersymmetric extension of the conformal fourth-order scalar operator (introduced originally by Fradkin and Tseytlin and also known as the "Paneitz operator" or "Riegert operator"), we derive a new representation for the nonlocal action generating the super-Weyl anomalies.
\end{abstract}




\section{Introduction}

The concept of super-Weyl transformations [1, 2] in the framework of the Wess-Zumino formulation [3, 4] for $4 \mathrm{D} \mathcal{N}=1$ supergravity is a generalization of the ordinary Weyl transformations. The property of a locally supersymmetric theory to be super-Weyl invariant can naturally be recast in terms of the Ferrara-Zumino supercurrent multiplet [5] which contains the energy-momentum tensor and the spinor supersymmetry current along with some other components. It is pertinent for our subsequent discussion to elaborate on this point in some more detail.

The Ferrara-Zumino multiplet is realized in terms of two constrained superfields: a real vector $T_{a}$ known as the supercurrent and a covariantly chiral scalar $T, \overline{\mathcal{D}}_{\dot{\alpha}} T=0$, called the supertrace (also known as the anomaly multiplet). The supercurrent is the source of supergravity [6, 17, 8]. Let $S[\chi, H, \varphi, \bar{\varphi}]$ be the action of matter superfields $\chi^{i}$ coupled to background supergravity. The supergravity multiplet is fully described in terms of the corresponding prepotentials $H^{m}, \varphi$ and $\bar{\varphi}$ [9, 10], of which the gravitational superfield [6] $H^{m}$ is real and the conformal compensator [9] $\varphi$ is chiral. The supercurrent and the supertrace originate as variational derivatives of the matter action with respect to the supergravity prepotential. Specifically, it turns out that

$$
\begin{aligned}
T_{a} & =\frac{\Delta}{\Delta H^{a}} S[\chi, H, \varphi, \bar{\varphi}], \\
T & =\left.\frac{\delta}{\delta \sigma} S\left[\chi, H, \mathrm{e}^{\sigma} \varphi, \mathrm{e}^{\bar{\sigma}} \bar{\varphi}\right]\right|_{\sigma=\bar{\sigma}=0}, \quad \overline{\mathcal{D}}_{\dot{\alpha}} \sigma=0,
\end{aligned}
$$

where $\sigma$ is an arbitrary covariantly chiral scalar superfield. Here $\Delta / \Delta H^{a}$ denotes a covariantized variational derivative with respect to the gravitational superfield, see [11, 12] for pedagogical reviews. If the matter fields are chosen to obey their equations of motion, $\delta S / \delta \chi^{i}=0$, the condition that the matter action is locally supersymmetric is expressed as the conservation equation

$$
\overline{\mathcal{D}}^{\dot{\alpha}} T_{\alpha \dot{\alpha}}+\frac{2}{3} \mathcal{D}_{\alpha} T=0
$$

A super-Weyl transformation [1] is a local rescaling of the chiral compensator [2], $\varphi \rightarrow \varphi^{\prime}=\mathrm{e}^{\sigma} \varphi$, accompanied by a certain transformation of the matter superfields of the form $\chi^{i} \rightarrow \chi^{i}=\mathrm{e}^{-d_{(+)} \sigma-d_{(-)} \bar{\sigma}} \chi^{i}$, for some parameters $d_{( \pm)}$(with the gravitational superfield $H^{m}$ being super-Weyl inert). If the matter fields in (1.1) are chosen to satisfy the equations of motion $\delta S / \delta \chi^{i}=0$, it follows from (1.1b) that $T$ determines the superWeyl variation of the action. Without imposing the matter equations of motion, the 
super-Weyl variation of the action is

$$
\delta_{\sigma} S=\int \mathrm{d}^{4} x \mathrm{~d}^{2} \theta \mathcal{E} \sigma T+\text { c.c. }+\int \delta_{\sigma} \chi^{i} \cdot \frac{\delta S}{\delta \chi^{i}},
$$

where $\mathcal{E}=\varphi^{3}$ denotes the chiral integration measure (see, e.g., [11, 12] for more details). If the theory is super-Weyl invariant, then $T=0$ for the on-shell matter fields. In a rigid supersymmetric limit, when $H^{m} \rightarrow 0$ and $\varphi \rightarrow 1$, such a theory becomes superconformal.

In the quantum theory, integrating out the matter fields leads to the effective action $\Gamma[H, \varphi, \bar{\varphi}]$. The quantum supertrace is

$$
\langle T\rangle=\left.\frac{\delta}{\delta \sigma} \Gamma\left[H, \mathrm{e}^{\sigma} \varphi, \mathrm{e}^{\bar{\sigma}} \bar{\varphi}\right]\right|_{\sigma=\bar{\sigma}=0} .
$$

Generically, the super-Weyl symmetry is anomalous at the quantum level. This means that, if one starts from a classically super-Weyl invariant theory, then in general $\langle T\rangle$ turns out to be a non-zero local functional of the supergravity prepotentials, which cannot be eliminated by adding local counterterms to the effective action. According to the cohomological analysis given in [13], the general form of $\langle T\rangle$ in classically super-Weyl invariant theories is

$$
8 \pi^{2}\langle T\rangle=c W^{\alpha \beta \gamma} W_{\alpha \beta \gamma}-a \mathcal{G}+\frac{1}{16} h\left(\overline{\mathcal{D}}^{2}-4 R\right) \mathcal{D}^{2} R
$$

where $\mathcal{G}$ denotes the following chiral scalar:

$$
\mathcal{G}=W^{\alpha \beta \gamma} W_{\alpha \beta \gamma}-\frac{1}{4}\left(\overline{\mathcal{D}}^{2}-4 R\right)\left(G^{a} G_{a}+2 R \bar{R}\right) .
$$

It turns out that the functional [7] (with $E$ the full superspace integration measure)

$$
\int \mathrm{d}^{4} x \mathrm{~d}^{2} \theta \mathcal{E} \mathcal{G}=\int \mathrm{d}^{4} x \mathrm{~d}^{2} \theta \mathcal{E} W^{\alpha \beta \gamma} W_{\alpha \beta \gamma}+\int \mathrm{d}^{4} x \mathrm{~d}^{2} \theta \mathrm{d}^{2} \bar{\theta} E\left(G^{a} G_{a}+2 R \bar{R}\right)
$$

is a topological invariant (see [14, 12] for the proof), which is related to the difference of the Gauss-Bonnet and Pontryagin invariants. The coefficients $a$ and $c$ in (1.5) are of nontrivial significance in general superconformal field theories, see [15, 16, 17, 18] and references therein. On the other hand, the coefficient $h$ may be completely arbitrary, for its value may be freely changed by adding to the effective action a finite local counterterm proportional to

$$
\int \mathrm{d}^{4} x \mathrm{~d}^{2} \theta \mathrm{d}^{2} \bar{\theta} E R \bar{R}
$$

The explicit calculation of the anomalous supertrace (1.5) for the chiral and vector multiplets was carried out in [19] (see [12] for a review). This calculation made use of 
the super- $a_{2}$ (or, equivalently, super- $b_{4}$ ) coefficients computed by McArthur [20] (for the chiral multiplet) using the superspace normal coordinates [21] and independently in [19] using the superfield Schwinger-DeWitt technique. Given the anomalous supertrace (1.5), it becomes possible to look for a nonlocal effective action that generates the anomaly. Such an action was derived in [22] (see [12] for a review). This was done with the aid of a composite chiral scalar $\Omega$, introduced originally in [23], which obeys the massless equation

$$
\left(\mathcal{D}^{2}-4 \bar{R}\right) \Omega=0, \quad \overline{\mathcal{D}}_{\dot{\alpha}} \Omega=0
$$

and possesses the super-Weyl transformation law1

$$
\Omega \rightarrow \Omega^{\prime}=\mathrm{e}^{-\sigma} \Omega
$$

The explicit expression for $\Omega$ is

$$
\Omega=1+\frac{1}{4 \square_{+}}\left(\overline{\mathcal{D}}^{2}-4 R\right) \bar{R}
$$

where $\square_{+}$denotes the chiral d'Alembertian defined by $\square_{+} \phi=\frac{1}{16}\left(\overline{\mathcal{D}}^{2}-4 R\right)\left(\mathcal{D}^{2}-4 \bar{R}\right) \phi$, for any covariantly chiral scalar $\phi$.

Conceptually, the chiral superfield $\Omega$ is a supersymmetric analogue of the composite scalar field $\omega=1+\frac{1}{6}\left(\square-\frac{1}{6} \mathcal{R}\right)^{-1} \mathcal{R}$, with $\mathcal{R}$ the scalar curvature, introduced by Fradkin and Vilkovisky [24]. The scalar field $\omega$ was used by Fradkin and Tseytlin [25] to integrate the ordinary Weyl (or conformal) anomalies (see [26, 27, 28, 29] and references therein). So the approach of [22] may be thought of as a supersymmetric extension of that given in [25]. In the case of the Weyl anomaly, there exists an alternative method [30] to construct a nonlocal action generating the anomaly 2 It makes use of the conformal fourth-order scalar operator

$$
\Delta_{0}=\square \square+\mathcal{D}^{a}\left(2 \mathcal{R}_{a b} \mathcal{D}^{b}-\frac{2}{3} \mathcal{R} \mathcal{D}_{a}\right)
$$

discovered by Fradkin and Tseytlin] in 1981 [34] and re-discovered by Paneitz in 1983 [35] 4

\footnotetext{
${ }^{1}$ Using the super-Weyl transformation of the antichiral torsion $\bar{R}, \bar{R} \rightarrow \bar{R}^{\prime}=-\frac{1}{4} \mathrm{e}^{-2 \bar{\sigma}}\left(\mathcal{D}^{2}-4 \bar{R}\right) \mathrm{e}^{\sigma}$, one can see that $\Omega$ defines a super-Weyl transformation such that $\bar{R}^{\prime}=0$ and $R^{\prime}=0$.

${ }^{2}$ Nonlocal actions for Weyl anomalies in higher dimensions were studied in [31, 32, 33].

${ }^{3}$ Fradkin and Tseytlin [34 also constructed conformal operators $\Delta_{s}$ for fields of different spin.

${ }^{4}$ In the mathematics literature, this operator is known as the Paneitz operator. In the first preprint version of our paper, it was called "the Paneitz-Riegert operator" since the same operator had appeared in [30] and we were not aware of the earlier work by Fradkin and Tseytlin [34].
} 
It was mentioned in [22] that the technique of [30] was not "directly applicable for the anomaly integration in curved superspace." The reason for that was the non-existence of a super-Weyl covariant chiral d'Alembertian $\widehat{\square}_{+}$such that the functional

$$
\int \mathrm{d}^{4} x \mathrm{~d}^{2} \theta \mathrm{d}^{2} \bar{\theta} E \bar{\Phi} \widehat{\square}_{+} \Psi=\frac{1}{16} \int \mathrm{d}^{4} x \mathrm{~d}^{2} \theta \mathrm{d}^{2} \bar{\theta} E\left(\overline{\mathcal{D}}^{2} \bar{\Phi}\right) \mathcal{D}^{2} \Psi+\ldots
$$

is super-Weyl invariant for any super-Weyl inert chiral scalars $\Phi$ and $\Psi$ (the ellipsis on the right of (1.13) denotes terms with two or fewer spinor derivatives). The component counterpart of the operator in (1.13) is fourth-order in vector derivatives.

A way out has only recently been found in Ref. [36] which provided the required supersymmetric extension of the Fradkin-Tseytlin $\Delta_{0}$ operator (using the novel superspace formulation [37] for $\mathcal{N}=1$ conformal supergravity). Here we will use this operator to derive a new representation for the nonlocal action generating the super-Weyl anomaly.

This paper is organized as follows. In section 2, we describe the properties of the supersymmetric Fradkin-Tseytlin operator. In section 3, we derive the nonlocal effective action generating the super-Weyl anomaly. Concluding comments are given in section 4 . A brief summary of the Wess-Zumino superspace geometry, following the notation and conventions of [12], is given in the appendix.

\section{The supersymmetric Fradkin-Tseytlin operator}

In this section we give a detailed derivation of the $\mathcal{N}=1$ super-Weyl covariant operator which was introduced in [36] as the supersymmetric extension of the Fradkin-Tseytlin operator (1.12).

As is well known, the algebra of covariant derivatives (A.3) is invariant under the infinitesimal super-Weyl transformation [1] associated with a chiral parameter $\sigma, \overline{\mathcal{D}}_{\dot{\alpha}} \sigma=0$, and its complex conjugate $\bar{\sigma}$,

$$
\begin{aligned}
\delta_{\sigma} \mathcal{D}_{\alpha}= & \left(\frac{1}{2} \sigma-\bar{\sigma}\right) \mathcal{D}_{\alpha}-\left(\mathcal{D}^{\beta} \sigma\right) M_{\alpha \beta} \\
\delta_{\sigma} \overline{\mathcal{D}}_{\dot{\alpha}}= & \left(\frac{1}{2} \bar{\sigma}-\sigma\right) \overline{\mathcal{D}}_{\dot{\alpha}}-\left(\overline{\mathcal{D}}^{\dot{\beta}} \bar{\sigma}\right) \bar{M}_{\dot{\alpha} \dot{\beta}} \\
\delta_{\sigma} \mathcal{D}_{\alpha \dot{\alpha}}= & -\frac{1}{2}(\sigma+\bar{\sigma}) \mathcal{D}_{\alpha \dot{\alpha}}-\frac{\mathrm{i}}{2}\left(\overline{\mathcal{D}}_{\dot{\alpha}} \bar{\sigma}\right) \mathcal{D}_{\alpha}-\frac{\mathrm{i}}{2}\left(\mathcal{D}_{\alpha} \sigma\right) \overline{\mathcal{D}}_{\dot{\alpha}} \\
& -\left(\mathcal{D}^{\beta}{ }_{\dot{\alpha}} \sigma\right) M_{\alpha \beta}-\left(\mathcal{D}_{\alpha}{ }^{\dot{\beta}} \bar{\sigma}\right) \bar{M}_{\dot{\alpha} \dot{\beta}}
\end{aligned}
$$

\footnotetext{
${ }^{5}$ Given a chiral d'Alembertian $\widehat{\square}_{+}$such that $\widehat{\square}_{+} \Psi$ is covariantly chiral for any covariantly chiral scalar $\Psi$, the differential part of $\widehat{\square}_{+}$is uniquely fixed [19, $\widehat{\square}_{+}=\mathcal{D}^{a} \mathcal{D}_{a}+\frac{1}{4} R \mathcal{D}^{2}+\mathrm{i} G^{a} \mathcal{D}_{a}+\frac{1}{4}\left(\mathcal{D}^{\alpha} R\right) \mathcal{D}_{\alpha}+\mathcal{P}$, where the scalar superfield $\mathcal{P}$ is covariantly chiral. The only free parameter in $\widehat{\square}_{+}$is the chiral scalar $\mathcal{P}$.
} 
provided the torsion tensors transform 6 as follows:

$$
\begin{aligned}
\delta_{\sigma} R & =-2 \sigma R-\frac{1}{4}\left(\overline{\mathcal{D}}^{2}-4 R\right) \bar{\sigma}=(\bar{\sigma}-2 \sigma) R-\frac{1}{4} \overline{\mathcal{D}}^{2} \bar{\sigma}, \\
\delta_{\sigma} G_{\alpha \dot{\alpha}} & =-\frac{1}{2}(\sigma+\bar{\sigma}) G_{\alpha \dot{\alpha}}+\mathrm{i} \mathcal{D}_{\alpha \dot{\alpha}}(\bar{\sigma}-\sigma), \\
\delta_{\sigma} W_{\alpha \beta \gamma} & =-\frac{3}{2} \sigma W_{\alpha \beta \gamma} .
\end{aligned}
$$

Let $\bar{\Phi}$ be a covariantly antichiral scalar, $\mathcal{D}_{\alpha} \bar{\Phi}=0$, invariant under the super-Weyl transformations, $\delta_{\sigma} \bar{\Phi}=0$. It is an instructive exercise to check, using the algebra of covariant derivatives given in the Appendix, the following super-Weyl transformation law:

$$
\begin{aligned}
\delta_{\sigma}\left\{\mathcal{D}^{2} \overline{\mathcal{D}}^{2} \bar{\Phi}+8 \mathcal{D}^{\alpha}\left(G_{\alpha \dot{\alpha}} \overline{\mathcal{D}}^{\dot{\alpha}} \bar{\Phi}\right)\right\}= & -(\sigma+\bar{\sigma})\left\{\mathcal{D}^{2} \overline{\mathcal{D}}^{2} \bar{\Phi}+8 \mathcal{D}^{\alpha}\left(G_{\alpha \dot{\alpha}} \overline{\mathcal{D}}^{\dot{\alpha}} \bar{\Phi}\right)\right\} \\
& -2 \overline{\mathcal{D}}_{\dot{\alpha}}\left\{\left(\overline{\mathcal{D}}^{\dot{\alpha}} \bar{\Phi}\right) \mathcal{D}^{2} \sigma+4 \mathrm{i}\left(\mathcal{D}^{\alpha \dot{\alpha}} \bar{\Phi}\right) \mathcal{D}_{\alpha} \sigma\right\}
\end{aligned}
$$

To simplify the calculation, it is advantageous to make use of the identity

$$
\delta_{\sigma} \mathcal{D}^{2}=(\sigma-2 \bar{\sigma}) \mathcal{D}^{2}+2\left(\mathcal{D}^{\alpha} \sigma\right) \mathcal{D}_{\alpha}+\ldots
$$

where the ellipsis stands for all terms which involve the Lorentz generators and annihilate scalars. It follows from (2.3) that the operator $\Delta$ defined by

$$
\Delta \bar{\Phi}:=-\frac{1}{64}\left(\overline{\mathcal{D}}^{2}-4 R\right)\left\{\mathcal{D}^{2} \overline{\mathcal{D}}^{2} \bar{\Phi}+8 \mathcal{D}^{\alpha}\left(G_{\alpha \dot{\alpha}} \overline{\mathcal{D}}^{\dot{\alpha}} \bar{\Phi}\right)\right\}, \quad \overline{\mathcal{D}}_{\dot{\alpha}} \Delta \bar{\Phi}=0
$$

has the super-Weyl transformation law

$$
\delta_{\sigma} \Delta \bar{\Phi}=-3 \sigma \Delta \bar{\Phi}
$$

We also introduce the conjugate operator

$$
\bar{\Delta} \Phi:=-\frac{1}{64}\left(\mathcal{D}^{2}-4 \bar{R}\right)\left\{\overline{\mathcal{D}}^{2} \mathcal{D}^{2} \Phi-8 \overline{\mathcal{D}}^{\dot{\alpha}}\left(G_{\alpha \dot{\alpha}} \mathcal{D}^{\alpha} \Phi\right)\right\}, \quad \mathcal{D}_{\alpha} \bar{\Delta} \Phi=0 .
$$

For any covariantly chiral scalars $\Phi$ and $\Psi$, it holds that

$$
\begin{aligned}
\int \mathrm{d}^{4} x \mathrm{~d}^{2} \theta \mathcal{E} \Psi \Delta \bar{\Phi} & =\int \mathrm{d}^{4} x \mathrm{~d}^{2} \bar{\theta} \overline{\mathcal{E}} \bar{\Phi} \bar{\Delta} \Psi \\
& =\frac{1}{16} \int \mathrm{d}^{4} x \mathrm{~d}^{2} \theta \mathrm{d}^{2} \bar{\theta} E\left\{\left(\mathcal{D}^{2} \Psi\right) \overline{\mathcal{D}}^{2} \bar{\Phi}-8\left(\mathcal{D}^{\alpha} \Psi\right) G_{\alpha \dot{\alpha}} \overline{\mathcal{D}}^{\dot{\alpha}} \bar{\Phi}\right\}
\end{aligned}
$$

\footnotetext{
${ }^{6}$ The super-Weyl transformation of $G_{\alpha \dot{\alpha}}$ given in [12, eq. (5.5.14), contains a typo.
} 
In accordance with (2.6) , this functional is super-Weyl invariant provided the chiral scalars $\Phi$ and $\Psi$ are inert under the super-Weyl transformations. In the case that $\Phi=\Psi$, the functional (2.8) is real. Its component expression was given in [38] up to terms involving the gravitino.

The functional (2.8) bears some resemblance to (1.13) since both possess the same flat space limit, but the operators underlying these two constructions are quite different. The chiral d'Alembertian, which can be written $\widehat{\square}_{+}=\frac{1}{16}\left(\overline{\mathcal{D}}^{2}-4 R\right) \mathcal{D}^{2}+\mathcal{P}$, is a dimension2 operator mapping a chiral multiplet to another chiral multiplet; however, it cannot be made super-Weyl covariant for any choice of the chiral function $\mathcal{P}$, which was the point made in [22]. In contrast, the operator $\Delta$ underlying (2.8) is a dimension-3 superWeyl covariant operator; it acts on a weight-zero antichiral to yield a weight-three chiral superfield. The overall chiral projector in (2.5) is critical for achieving this manifest superWeyl covariance, because the quantity in braces in (2.5) is neither super-Weyl covariant nor of definite chirality by itself. The resulting super-Weyl covariance of the functional (2.8) is absolutely critical for lifting the Fradkin-Tseytlin operator to superspace and for enabling the construction which follows.

\section{Nonlocal effective action}

We now turn to deriving a nonlocal action that generates the super-Weyl anomaly (1.5). We begin by introducing several important building blocks.

First, we require the super-Weyl transformations of some composite expressions involving the torsion superfields. Using eq. (2.2), one can show

$$
\begin{aligned}
\delta_{\sigma}\left(G^{a} G_{a}+2 R \bar{R}\right)= & -(\sigma+\bar{\sigma})\left(G^{a} G_{a}+2 R \bar{R}\right)-\frac{1}{2} \overline{\mathcal{D}}_{\dot{\alpha}}\left(\bar{R} \overline{\mathcal{D}}^{\dot{\alpha}} \bar{\sigma}\right)-\frac{1}{2} \mathcal{D}^{\alpha}\left(R \mathcal{D}_{\alpha} \sigma\right) \\
& -\frac{1}{2} \overline{\mathcal{D}}^{\dot{\alpha}}\left(G_{\alpha \dot{\alpha}} \mathcal{D}^{\alpha} \sigma\right)+\frac{1}{2} \mathcal{D}^{\alpha}\left(G_{\alpha \dot{\alpha}} \overline{\mathcal{D}}^{\dot{\alpha}} \bar{\sigma}\right)
\end{aligned}
$$

which guarantees that the functional

$$
\int \mathrm{d}^{4} x \mathrm{~d}^{2} \theta \mathrm{d}^{2} \bar{\theta} E\left(G^{a} G_{a}+2 R \bar{R}\right)
$$

is super-Weyl invariant [1]. Making use of the super-Weyl variation

$$
\delta_{\sigma}\left(\mathcal{D}^{2} R\right)=-(\sigma+\bar{\sigma}) \mathcal{D}^{2} R-2 \mathcal{D}^{\alpha}\left(R \mathcal{D}_{\alpha} \sigma\right)-\frac{1}{4} \mathcal{D}^{2} \overline{\mathcal{D}}^{2} \bar{\sigma}
$$


we further observe that

$$
\begin{aligned}
\delta_{\sigma}\left\{G^{a} G_{a}+2 R \bar{R}\right. & \left.-\frac{1}{4} \mathcal{D}^{2} R\right\}=-(\sigma+\bar{\sigma})\left\{G^{a} G_{a}+2 R \bar{R}-\frac{1}{4} \mathcal{D}^{2} R\right\} \\
& +\frac{1}{16} \mathcal{D}^{2} \overline{\mathcal{D}}^{2} \bar{\sigma}+\frac{1}{2} \mathcal{D}^{\alpha}\left(G_{\alpha \dot{\alpha}} \overline{\mathcal{D}}^{\dot{\alpha}} \bar{\sigma}\right)+\frac{1}{2} \overline{\mathcal{D}}^{\dot{\alpha}}\left(\bar{R} \overline{\mathcal{D}}_{\dot{\alpha}} \bar{\sigma}-G_{\alpha \dot{\alpha}} \mathcal{D}^{\alpha} \sigma\right)
\end{aligned}
$$

Now we introduce the composite chiral scalar

$$
\Xi:=-\frac{1}{4}\left(\overline{\mathcal{D}}^{2}-4 R\right)\left\{G^{a} G_{a}+2 R \bar{R}-\frac{1}{4} \mathcal{D}^{2} R\right\}
$$

and verify that

$$
\delta_{\sigma} \Xi=-3 \sigma \Xi+\Delta \bar{\sigma} .
$$

As a next step, we introduce two scalar Green's functions $G_{+-}\left(z, z^{\prime}\right)$ and $G_{-+}\left(z, z^{\prime}\right)$ that are related to each other by the rule

$$
G_{+-}\left(z, z^{\prime}\right)=G_{-+}\left(z^{\prime}, z\right)
$$

and obey the following conditions:

(i) the two-point function $G_{-+}\left(z, z^{\prime}\right)$ is covariantly antichiral in $z$ and chiral in $z^{\prime}$,

$$
\mathcal{D}_{\alpha} G_{-+}\left(z, z^{\prime}\right)=0, \quad \overline{\mathcal{D}}_{\dot{\alpha}}^{\prime} G_{-+}\left(z, z^{\prime}\right)=0 ;
$$

(ii) the two-point function $G_{-+}\left(z, z^{\prime}\right)$ satisfies the differential equation

$$
\Delta G_{-+}\left(z, z^{\prime}\right)=\delta_{+}\left(z, z^{\prime}\right) .
$$

Here we have used the chiral delta-function

$$
\delta_{+}\left(z, z^{\prime}\right):=-\frac{1}{4}\left(\overline{\mathcal{D}}^{2}-4 R\right) E^{-1} \delta^{4}\left(x-x^{\prime}\right) \delta^{2}\left(\theta-\theta^{\prime}\right) \delta^{2}\left(\bar{\theta}-\bar{\theta}^{\prime}\right),
$$

which is covariantly chiral with respect to each of its arguments,

$$
\overline{\mathcal{D}}_{\dot{\alpha}} \delta_{+}\left(z, z^{\prime}\right)=0, \quad \overline{\mathcal{D}}_{\dot{\alpha}}^{\prime} \delta_{+}\left(z, z^{\prime}\right)=0 .
$$

Under a finite super-Weyl transformation, the delta-function $\delta_{+}\left(z, z^{\prime}\right)$ can be seen to change as

$$
\delta_{+}\left(z, z^{\prime}\right) \rightarrow \mathrm{e}^{-3 \sigma} \delta_{+}\left(z, z^{\prime}\right) .
$$

It follows from the relations (2.6), (3.9) and (3.12) that the Green's function $G_{-+}\left(z, z^{\prime}\right)$ is super-Weyl invariant, as is $G_{+-}\left(z, z^{\prime}\right)$. 
Finally, it is useful to introduce a condensed notation for the chiral and antichiral integration measures:

$$
\int \mathrm{d} \mu_{+}:=\int \mathrm{d}^{4} x \mathrm{~d}^{2} \theta \mathcal{E}, \quad \int \mathrm{d} \mu_{-}:=\int \mathrm{d}^{4} x \mathrm{~d}^{2} \bar{\theta} \overline{\mathcal{E}}
$$

With the building blocks given above, let us consider two nonlocal real functionals

$$
\begin{aligned}
& \mathcal{F}_{1}=\int \mathrm{d} \mu_{+} \int \mathrm{d} \mu_{-}^{\prime} \Xi(z) G_{+-}\left(z, z^{\prime}\right) \bar{\Xi}\left(z^{\prime}\right), \\
& \mathcal{F}_{2}=\int \mathrm{d} \mu_{+} \int \mathrm{d} \mu_{-}^{\prime} W^{2}(z) G_{+-}\left(z, z^{\prime}\right) \bar{\Xi}\left(z^{\prime}\right)+\text { c.c. },
\end{aligned}
$$

with $W^{2}=W^{\alpha \beta \gamma} W_{\alpha \beta \gamma}$. The super-Weyl variations of these functionals are

$$
\begin{aligned}
\delta_{\sigma} \mathcal{F}_{1} & =\int \mathrm{d} \mu_{+} \sigma \Xi+\text { c.c. }, \\
\delta_{\sigma} \mathcal{F}_{2} & =\int \mathrm{d} \mu_{+} \sigma W^{2}+\text { c.c. }
\end{aligned}
$$

We also note that the super-Weyl variation of the local functional (1.8) is

$$
\delta_{\sigma} \int \mathrm{d}^{4} x \mathrm{~d}^{2} \theta \mathrm{d}^{2} \bar{\theta} E R \bar{R}=\frac{1}{16} \int \mathrm{d} \mu_{+} \sigma\left(\overline{\mathcal{D}}^{2}-4 R\right) \mathcal{D}^{2} R+\text { c.c. }
$$

It follows from these relations that the effective action can be chosen in the form:

$$
\begin{aligned}
8 \pi^{2} \Gamma & =(c-a) \int \mathrm{d} \mu_{+} \int \mathrm{d} \mu_{-}^{\prime} W^{2}(z) G_{+-}\left(z, z^{\prime}\right) \bar{\Xi}\left(z^{\prime}\right)+\text { c.c. } \\
& -a \int \mathrm{d} \mu_{+} \int \mathrm{d} \mu_{-}^{\prime} \Xi(z) G_{+-}\left(z, z^{\prime}\right) \bar{\Xi}\left(z^{\prime}\right)+(h+a) \int \mathrm{d}^{4} x \mathrm{~d}^{2} \theta \mathrm{d}^{2} \bar{\theta} E R \bar{R} .
\end{aligned}
$$

\section{Concluding comments}

The nonlocal action (3.19) is one of the main results of our paper. It provides the generalization of Riegert's construction 730 to $\mathcal{N}=1$ supergravity. The crucial property of $\Gamma$ is that the corresponding supertrace $\langle T\rangle$, eq. (1.4), coincides with the super-Weyl anomaly (1.5). The same anomaly is generated by the effective action derived twenty five years ago in [22] (see eq. (14) in [22]). The effective action derived in [22] differs from

\footnotetext{
${ }^{7}$ Several papers [39, 32, 33] pointed out certain pathological properties of the nonlocal action derived in [30]. More recently, this issue has been reconsidered in Ref. [40] which "rehabilitated" [30]. Here we are only interested in the formal functional structure of the effective action.
} 
(3.19) by a super-Weyl invariant contribution that depends only on the gravitational superfield and therefore vanishes in any conformally flat superspace.

In general, the super-Weyl anomaly may include not only the purely supergravity sector (1.5), but also an additional contribution coming from a background vector supermultiplet, which is proportional to $\operatorname{tr}\left(W^{\alpha} W_{\alpha}\right)$, with $W_{\alpha}$ the covariantly chiral field strength. Such a contribution is taken into account by an additional correction to the effective action (3.19) obtained by the replacement

$$
W^{\alpha \beta \gamma} W_{\alpha \beta \gamma} \rightarrow \operatorname{tr}\left(W^{\alpha} W_{\alpha}\right)
$$

The functional (2.8) is super-Weyl invariant for arbitrary super-Weyl inert chiral scalars $\Phi$ and $\Psi$. In a locally supersymmetric theory, these chiral scalars may be chosen to be some of the dynamical variables or composite objects. Thus (2.8) generates a nontrivial higher derivative term in $\mathcal{N}=1$ supergravity.

Because the functional (2.8) is super-Weyl invariant, it is independent of the chiral compensator $\varphi$ and its conjugate. Therefore, this functional can naturally be described within any off-shell version of $\mathcal{N}=1$ supergravity. As is known, any off-shell formulation for $\mathcal{N}=1$ supergravity can be realized by coupling a certain compensator to $\mathrm{U}(1)$ superspace [41] (see [11] for a review), which gauges not only the Lorentz group but the $\mathrm{U}(1) R$-symmetry group as well 8 Using the same conventions as in [42], one finds that the operator $\Delta$ in $\mathrm{U}(1)$ superspace is given by

$$
\Delta \bar{\Phi}:=-\frac{1}{64}\left(\overline{\mathcal{D}}^{2}-4 R\right)\left\{\mathcal{D}^{2} \overline{\mathcal{D}}^{2} \bar{\Phi}+8 \mathcal{D}^{\alpha}\left(G_{\alpha \dot{\alpha}} \overline{\mathcal{D}}^{\dot{\alpha}} \bar{\Phi}\right)+\frac{16}{3} \bar{X}_{\dot{\alpha}} \overline{\mathcal{D}}^{\dot{\alpha}} \bar{\Phi}\right\}, \quad \overline{\mathcal{D}}_{\dot{\alpha}} \Delta \bar{\Phi}=0,
$$

and obeys the super-Weyl transformation

$$
\delta_{\Lambda} \Delta \bar{\Phi}=-3 \Lambda \Delta \bar{\Phi}
$$

where $\Lambda$ is a real unconstrained super-Weyl transformation parameter 9 In $U(1)$ superspace the functional (2.8) turns into

$$
\begin{aligned}
& \int \mathrm{d}^{4} x \mathrm{~d}^{2} \theta \mathcal{E} \Psi \Delta \bar{\Phi}=\int \mathrm{d}^{4} x \mathrm{~d}^{2} \bar{\theta} \overline{\mathcal{E}} \bar{\Phi} \bar{\Delta} \Psi \\
& =\frac{1}{16} \int \mathrm{d}^{4} x \mathrm{~d}^{2} \theta \mathrm{d}^{2} \bar{\theta} E\left\{\left(\mathcal{D}^{2} \Psi\right) \overline{\mathcal{D}}^{2} \bar{\Phi}-8\left(\mathcal{D}^{\alpha} \Psi\right) G_{\alpha \dot{\alpha}} \overline{\mathcal{D}}^{\dot{\alpha}} \bar{\Phi}-\frac{16}{3}\left(\overline{\mathcal{D}}_{\dot{\alpha}} \bar{X}^{\dot{\alpha}}\right) \Psi \bar{\Phi}\right\} .
\end{aligned}
$$

\footnotetext{
${ }^{8}$ In its turn, $\mathrm{U}(1)$ superspace [41] is a gauged-fixed version of conformal superspace [37].

${ }^{9}$ We have flipped the sign of $\Lambda$ relative to [42].
} 
This functional is real when $\Psi=\Phi$, keeping in mind that $\overline{\mathcal{D}}_{\dot{\alpha}} \bar{X}^{\dot{\alpha}}=\mathcal{D}^{\alpha} X_{\alpha}$. We note that the superspace formulation of new minimal supergravity can be derived from $\mathrm{U}(1)$ superspace by taking $R=0$, so the expressions (4.2) and (4.4) hold equally there.

As we already emphasized, the functional (2.8) is super-Weyl invariant under the condition that $\Phi$ and $\Psi$ are super-Weyl inert chiral scalars. Choosing different types of superfields makes it possible to construct alternative superconformal invariants. For instance, if $U$ and $V$ are super-Weyl inert real scalars, then the functional

$$
\frac{1}{16} \int \mathrm{d}^{4} x \mathrm{~d}^{2} \theta \mathrm{d}^{2} \bar{\theta} E U \mathcal{D}^{\alpha}\left(\overline{\mathcal{D}}^{2}-4 R\right) \mathcal{D}_{\alpha} V
$$

is super-Weyl invariant. In the case that $U=V$ is a dynamical superfield, this functional coincides with the action of an Abelian vector multiplet [3]. On the other hand, $U$ and $V$ may be composite, for instance $U=V=K\left(\phi^{I}, \bar{\phi}^{\bar{J}}\right)$, where $K$ is the Kähler potential of a Kähler manifold, and $\phi^{I}$ are super-Weyl inert chiral scalars. Then, the above functional generates a higher-derivative coupling which is invariant under arbitrary Kähler transformations $K(\phi, \bar{\phi}) \rightarrow K(\phi, \bar{\phi})+\Lambda(\phi)+\bar{\Lambda}(\bar{\phi})$, as well as under arbitrary holomorphic isometries of the target Kähler space.

The attempt to construct an $\mathcal{N}=1$ supersymmetric extension of the Fradkin-Tseytlin operator was made a few years ago by Grosse [43]. Unfortunately, the attempt was unsuccessful10 and this author concluded that "there is no superfield version of the Riegert operator for chiral fields of Weyl weight 0." Although he did consider the integrand in the second line of (2.8), he was not able to prove its super-Weyl invariance. Most likely, this is due to its rather complicated transformation law. We only discovered its invariance after first considering the associated higher-order operator $\Delta$ defined by (2.5), which proves to possess the remarkably simple transformation law (2.6). As demonstrated in [36], the most natural origin of $\Delta$ is within conformal superspace [37].

In this paper we only considered the Wess-Zumino formulation for $\mathcal{N}=1$ supergravity [3] which is characterized by the old minimal set of auxiliary fields [44]. As is well known, there exist two other off-shell formulations for $\mathcal{N}=1$ supergravity, the nonminimal [45, 10] and the new minimal [46]. In the case of non-minimal supergravity, the super-Weyl transformations were described by Siegel [2]. For $n=-1$ non-minimal supergravity, a superfield Fradkin-Tseytlin operator was constructed in [47]. Super-Weyl

\footnotetext{
${ }^{10}$ One of us (SMK) had incorrectly argued to Johannes Grosse in 2006 and Yu Nakayama in 2012 that a superfield version of $\Delta_{0}$ could not exist, based on the non-existence of the super-Weyl covariant d'Alembertian $\widehat{\square}_{+}$. While working on [36], it became apparent that the higher dimension operator $\Delta$ could play the required role in deriving a supersymmetric extension of Riegert's construction.
} 
transformations and trace anomalies in various off-shell versions for $\mathcal{N}=1$ supergravity have recently been discussed in [48].

\section{Acknowledgements:}

We thank Arkady Tseytlin for bringing the important references [34] to our attention. SMK is grateful to Stefan Theisen for a useful discussion and for pointing Ref. [40] out. The work of DB was supported by ERC Advanced Grant No. 246974, "Supersymmetry: a window to non-perturbative physics." The work of SMK was supported in part by the Australian Research Council, project No. DP1096372.

\section{A The Wess-Zumino superspace geometry}

In describing the Wess-Zumino superspace geometry (see [49] for a review), we mostly follow the notation and conventions of [12]11 In particular, the coordinates of $\mathcal{N}=$ 1 curved superspace $\mathcal{M}$ are denoted $z^{M}=\left(x^{m}, \theta^{\mu}, \bar{\theta}_{\dot{\mu}}\right)$. The superspace geometry is described by covariant derivatives of the form

$$
\mathcal{D}_{A}=\left(\mathcal{D}_{a}, \mathcal{D}_{\alpha}, \overline{\mathcal{D}}^{\dot{\alpha}}\right)=E_{A}+\Omega_{A}
$$

Here $E_{A}$ denotes the inverse vielbein, $E_{A}=E_{A}{ }^{M} \partial_{M}$, and $\Omega_{A}$ the Lorentz connection,

$$
\Omega_{A}=\frac{1}{2} \Omega_{A}^{b c} M_{b c}=\Omega_{A}{ }^{\beta \gamma} M_{\beta \gamma}+\Omega_{A}^{\dot{\beta} \dot{\gamma}} \bar{M}_{\dot{\beta} \dot{\gamma}}
$$

with $M_{b c} \Leftrightarrow\left(M_{\beta \gamma}, \bar{M}_{\dot{\beta} \dot{\gamma}}\right)$ the Lorentz generators. The covariant derivatives obey the following anti-commutation relations:

$$
\begin{aligned}
\left\{\mathcal{D}_{\alpha}, \overline{\mathcal{D}}_{\dot{\alpha}}\right\} & =-2 \mathrm{i} \mathcal{D}_{\alpha \dot{\alpha}}, \quad\left\{\mathcal{D}_{\alpha}, \mathcal{D}_{\beta}\right\}=-4 \bar{R} M_{\alpha \beta}, \quad\left\{\overline{\mathcal{D}}_{\dot{\alpha}}, \overline{\mathcal{D}}_{\dot{\beta}}\right\}=4 R \bar{M}_{\dot{\alpha} \dot{\beta}} \\
{\left[\overline{\mathcal{D}}_{\dot{\alpha}}, \mathcal{D}_{\beta \dot{\beta}}\right] } & =-\mathrm{i} \varepsilon_{\dot{\alpha} \dot{\beta}}\left(R \mathcal{D}_{\beta}+G_{\beta}^{\dot{\gamma}} \overline{\mathcal{D}}_{\dot{\gamma}}-\left(\overline{\mathcal{D}}^{\dot{\gamma}} G_{\beta} \dot{\delta}\right) \bar{M}_{\dot{\gamma} \dot{\delta}}+2 W_{\beta}^{\gamma \delta} M_{\gamma \delta}\right)-\mathrm{i}\left(\mathcal{D}_{\beta} R\right) \bar{M}_{\dot{\alpha} \dot{\beta}} \\
{\left[\mathcal{D}_{\alpha}, \mathcal{D}_{\beta \dot{\beta}}\right] } & =\mathrm{i} \varepsilon_{\alpha \beta}\left(\bar{R} \overline{\mathcal{D}}_{\dot{\beta}}+G_{\dot{\beta}}^{\gamma} \mathcal{D}_{\gamma}-\left(\mathcal{D}^{\gamma} G_{\dot{\beta}}^{\delta}\right) M_{\gamma \delta}+2 \bar{W}_{\dot{\beta}}^{\dot{\gamma} \dot{\delta}} \bar{M}_{\dot{\gamma} \dot{\delta}}\right)+\mathrm{i}\left(\overline{\mathcal{D}}_{\dot{\beta}} \bar{R}\right) M_{\alpha \beta}
\end{aligned}
$$

\footnotetext{
${ }^{11}$ These conventions are nearly identical to those of Wess and Bagger [49. To convert the notation of [12] to that of [49], one replaces $R \rightarrow 2 R, G_{\alpha \dot{\alpha}} \rightarrow 2 G_{\alpha \dot{\alpha}}$, and $W_{\alpha \beta \gamma} \rightarrow 2 W_{\alpha \beta \gamma}$. In addition, the vector derivative has to be changed by the rule $\mathcal{D}_{a} \rightarrow \mathcal{D}_{a}+\frac{1}{4} \varepsilon_{a b c d} G^{b} M^{c d}$, where $G_{a}$ corresponds to [12]. Finally, the spinor Lorentz generators $\left(\sigma_{a b}\right)_{\alpha}{ }^{\beta}$ and $\left(\tilde{\sigma}_{a b}\right)^{\dot{\alpha}}{ }_{\dot{\beta}}$ used in [12] have an extra minus sign as compared with [49, specifically $\sigma_{a b}=-\frac{1}{4}\left(\sigma_{a} \tilde{\sigma}_{b}-\sigma_{b} \tilde{\sigma}_{a}\right)$ and $\tilde{\sigma}_{a b}=-\frac{1}{4}\left(\tilde{\sigma}_{a} \sigma_{b}-\tilde{\sigma}_{b} \sigma_{a}\right)$. Unlike [12], in this paper the full superspace measure is denoted $E=\operatorname{Ber}\left(E_{M}{ }^{A}\right)$.
} 
The torsion tensors $R, G_{a}=\bar{G}_{a}$ and $W_{\alpha \beta \gamma}=W_{(\alpha \beta \gamma)}$ satisfy the Bianchi identities

$$
\begin{aligned}
\overline{\mathcal{D}}_{\dot{\alpha}} R & =0, \quad \overline{\mathcal{D}}_{\dot{\alpha}} W_{\alpha \beta \gamma}=0, \\
\overline{\mathcal{D}}^{\dot{\gamma}} G_{\alpha \dot{\gamma}} & =\mathcal{D}_{\alpha} R, \\
\mathcal{D}^{\gamma} W_{\alpha \beta \gamma} & =\mathrm{i} \mathcal{D}_{(\alpha} \dot{\gamma} G_{\beta) \dot{\gamma}} .
\end{aligned}
$$

\section{References}

[1] P. S. Howe and R. W. Tucker, "Scale invariance in superspace," Phys. Lett. B 80, 138 (1978).

[2] W. Siegel, "Superconformal invariance of superspace with nonminimal auxiliary fields," Phys. Lett. B 80, 224 (1979).

[3] J. Wess and B. Zumino, "Superfield Lagrangian for supergravity," Phys. Lett. B 74, 51 (1978).

[4] R. Grimm, J. Wess and B. Zumino, "A complete solution of the Bianchi identities in superspace," Nucl. Phys. B 152, 255 (1979).

[5] S. Ferrara and B. Zumino, "Transformation properties of the supercurrent," Nucl. Phys. B 87, 207 (1975).

[6] V. Ogievetsky and E. Sokatchev, "On vector superfield generated by supercurrent," Nucl. Phys. B 124, 309 (1977).

[7] S. Ferrara and B. Zumino, "Structure of linearized supergravity and conformal supergravity," Nucl. Phys. B 134, 301 (1978).

[8] W. Siegel, "A derivation of the supercurrent superfield," Harvard preprint HUTP-77/A089 (December, 1977).

[9] W. Siegel, "Solution to constraints in Wess-Zumino supergravity formalism," Nucl. Phys. B 142, 301 (1978).

[10] W. Siegel and S. J. Gates Jr. "Superfield supergravity," Nucl. Phys. B 147, 77 (1979).

[11] S. J. Gates Jr., M. T. Grisaru, M. Roček and W. Siegel, Superspace, or One Thousand and One Lessons in Supersymmetry, Benjamin/Cummings (Reading, MA), 1983, hep-th/0108200

[12] I. L. Buchbinder and S. M. Kuzenko, Ideas and methods of supersymmetry and supergravity: Or a walk through superspace, Bristol, UK: IOP (1998) 656 p.

[13] L. Bonora, P. Pasti and M. Tonin, "Cohomologies and anomalies in supersymmetric theories," Nucl. Phys. B 252, 458 (1985).

[14] I. L. Buchbinder and S. M. Kuzenko, Quantization of the classically equivalent theories in the superspace of simple supergravity and quantum equivalence, Nucl. Phys. B 308, 162 (1988).

[15] D. Anselmi, D. Z. Freedman, M. T. Grisaru and A. A. Johansen, "Nonperturbative formulas for central functions of supersymmetric gauge theories," Nucl. Phys. B 526, 543 (1998) hep-th/9708042. 
[16] H. Osborn, "N=1 superconformal symmetry in four-dimensional quantum field theory," Annals Phys. 272, 243 (1999) hep-th/9808041].

[17] A. Schwimmer and S. Theisen, "Spontaneous breaking of conformal invariance and trace anomaly matching," Nucl. Phys. B 847, 590 (2011) [arXiv:1011.0696 [hep-th]].

[18] Z. Komargodski and A. Schwimmer, "On renormalization group flows in four dimensions," JHEP 1112, 099 (2011) arXiv:1107.3987 [hep-th]].

[19] I. L. Buchbinder and S. M. Kuzenko, "Matter superfields in external supergravity: Green functions, effective action and superconformal anomalies," Nucl. Phys. B 274, 653 (1986).

[20] I. N. McArthur, "Super b(4) coefficients," Phys. Lett. B 128, 194 (1983); "Super b(4) coefficients in supergravity," Class. Quant. Grav. 1, 245 (1984).

[21] I. N. McArthur, "Superspace normal coordinates," Class. Quant. Grav. 1, 233 (1984).

[22] I. L. Buchbinder and S. M. Kuzenko, "Nonlocal action for supertrace anomalies in superspace of N=1 supergravity," Phys. Lett. B 202, 233 (1988).

[23] M. T. Grisaru, N. K. Nielsen, W. Siegel and D. Zanon, "Energy-momentum tensors, supercurrents, (super)traces and quantum equivalence," Nucl. Phys. B 247, 157 (1984).

[24] E. S. Fradkin and G. A. Vilkovisky, "Conformal off mass shell extension and elimination of conformal anomalies in quantum gravity," Phys. Lett. B 73, 209 (1978).

[25] E. S. Fradkin and A. A. Tseytlin, "Conformal anomaly in Weyl theory and anomaly free superconformal theories," Phys. Lett. B 134, 187 (1984).

[26] S. Deser, M. J. Duff and C. J. Isham, "Nonlocal conformal anomalies," Nucl. Phys. B 111, 45 (1976).

[27] M. J. Duff, “Observations on conformal anomalies," Nucl. Phys. B 125, 334 (1977).

[28] S. Deser and A. Schwimmer, "Geometric classification of conformal anomalies in arbitrary dimensions," Phys. Lett. B 309, 279 (1993) hep-th/9302047.

[29] M. J. Duff, "Twenty years of the Weyl anomaly," Class. Quant. Grav. 11, 1387 (1994) hep-th/9308075.

[30] R. J. Riegert, "A non-local action for the trace anomaly," Phys. Lett. B 134 (1984) 56.

[31] D. R. Karakhanian, R. P. Manvelyan and R. L. Mkrtchian, "Trace anomalies and cocycles of Weyl and diffeomorphism groups," Mod. Phys. Lett. A 11, 409 (1996) hep-th/9411068.

[32] S. Deser, "Conformal anomalies: Recent progress," Helv. Phys. Acta 69, 570 (1996) hep-th/9609138.

[33] S. Deser, "Closed form effective conformal anomaly actions in $D \geq 4$," Phys. Lett. B 479, 315 (2000) hep-th/9911129].

[34] E. S. Fradkin and A. A. Tseytlin, "Asymptotic freedom in extended conformal supergravities," Phys. Lett. B 110, 117 (1982); "One-loop beta function in conformal supergravities," Nucl. Phys. B 203, 157 (1982). 
[35] S. M. Paneitz, "A quartic conformally covariant differential operator for arbitrary pseudoRiemannian manifolds," MIT preprint, March 1983; published posthumously in: SIGMA 4 (2008), 036, arXiv:0803.4331 [math.DG]].

[36] D. Butter, B. de Wit, S. M. Kuzenko and I. Lodato, "New higher-derivative invariants in N=2 supergravity and the Gauss-Bonnet term," work in progress.

[37] D. Butter, "N=1 Conformal superspace in four dimensions," Annals Phys. 325, 1026 (2010) arXiv:0906.4399 [hep-th]].

[38] E. S. Fradkin and A. A. Tseytlin, "Conformal supergravity," Phys. Rept. 119 (1985) 233.

[39] J. Erdmenger and H. Osborn, "Conserved currents and the energy momentum tensor in conformally invariant theories for general dimensions," Nucl. Phys. B 483, 431 (1997) hep-th/9605009.

[40] S. Deser, "Conformal anomalies revisited: Closed form effective actions in $D \geq 4$," Nucl. Phys. Proc. Suppl. 88, 204 (2000).

[41] P. S. Howe, "Supergravity in superspace," Nucl. Phys. B 199, 309 (1982).

[42] D. Butter and S. M. Kuzenko, "A dual formulation of supergravity-matter theories," Nucl. Phys. B 854, 1 (2012).

[43] J. Grosse, "Quantum field theories coupled to supergravity: AdS/CFT and local couplings," Fortsch. Phys. 56, 183 (2008) arXiv:0711.0444 [hep-th]].

[44] K. S. Stelle and P. C. West, "Minimal auxiliary fields for supergravity," Phys. Lett. B 74, 330 (1978); S. Ferrara and P. van Nieuwenhuizen, "The auxiliary fields of supergravity," Phys. Lett. B 74, 333 (1978).

[45] P. Breitenlohner, "A geometric interpretation of local supersymmetry," Phys. Lett. B 67, 49 (1977); "Some invariant Lagrangians for local supersymmetry," Nucl. Phys. B124, 500 (1977).

[46] M. F. Sohnius and P. C. West, "An alternative minimal off-shell version of $\mathrm{N}=1$ supergravity," Phys. Lett. B 105, 353 (1981); "The tensor calculus and matter coupling of the alternative minimal auxiliary field formulation of $\mathrm{N}=1$ supergravity," Nucl. Phys. B 198, 493 (1982).

[47] R. P. Manvelyan, "SuperWeyl cocycle in $\mathrm{d}=4$ and superconformal invariant operator," Phys. Lett. B 373, 306 (1996) hep-th/9512045.

[48] L. Bonora and S. Giaccari, "Weyl transformations and trace anomalies in N=1, D=4 supergravities," arXiv:1305.7116 [hep-th].

[49] J. Wess and J. Bagger, Supersymmetry and Supergravity, Princeton University Press, Princeton, 1983 (Second Edition 1992). 